\title{
What is the role of revascularisation in ischaemic heart failure?
}

The concept of myocardial hibernation was first proposed in the 1980s, and cardiologists and cardiac surgeons have become familiar with the possibility of improving ventricular function by restoring myocardial blood flow to dysfunctional but viable areas of myocardium subtended by a stenotic coronary artery. Despite this, coronary revascularisation has not been widely adopted as a strategy to treat patients with ischaemic heart failure. Indeed it seems likely that most cases of "ischaemic" heart failure are never investigated with a view to revascularisation. Are cardiologists and cardiac surgeons underinvestigating and treating this group of patients or are they properly directing scarce resources away from an intellectually attractive but expensive and high risk management strategy without proved benefit? A number of important questions remain to be answered before revascularisation to improve ventricular function can be considered as a standard treatment strategy for these patients.

- What proportion of patients with heart failure caused by coronary artery disease have potentially recoverable left ventricular dysfunction?

- How accurate are the techniques currently used to differentiate hibernating myocardium from irreversibly damaged tissue?

- How much viable myocardium is required for revascularisation to confer clinical benefit?

- Can coronary revascularisation be performed at acceptable risk in patients with poor ventricular function but viable myocardium?

- Most importantly, does revascularisation of hibernating myocardium result in sustained symptomatic and functional improvement as well as improvement in prognosis compared to continued optimal medical treatment?

What is the prevalence of hibernating myocardium? The prevalence of substantial viable myocardium and ultimately of recoverable left ventricular dysfunction in patients with coronary artery disease is unknown. Studies to date suggest that between $22 \%$ and $57 \%$ of dysfunctional left ventricular segments will have improvement in contractile function following coronary artery bypass graft (CABG), ${ }^{1-4}$ and more than $50 \%$ of patients with severe left ventricular dysfunction (ejection fraction $<35 \%$ ) enrolled in studies have shown clinically meaningful improvement in left ventricular function. ${ }^{35}$ These data are encouraging but come from highly selected patients.

\section{Identification of hibernating myocardium in heart failure}

Three techniques are commonly used to assess myocardial viability: dobutamine echocardiography, thallium-201 scintigraphy, and positron emission tomography (PET) with $\left[{ }^{18} \mathrm{~F}\right]$ 2-fluoro-2-deoxy-D-glucose (FDG). These methods probe different mechanisms associated with myocyte viability - preserved uptake of exogenous glucose for PET, sarcolemmal integrity for thallium-201, and the presence of inotropic contractile reserve for dobutamine echocardiography. In patients with normal or moderately impaired left ventricular function their predictive value for identification of recoverable left ventricular dysfunction (hibernating myocardium) appears to be similar (positive predictive value $69-83 \%$, negative predictive value $81-90 \%) .^{6}$ However, there are few data on patients with severe left ventricular dysfunction and clinical heart failure in whom the choice of management strategy (medical treatment, transplantation or revascularisation) is difficult, not least because the risks of CABG are high. Published studies have mostly included patients with angina in whom a decision to revascularise can be made on conventional clinical criteria. Thus the ability of these techniques to influence clinical decision making in patients with heart failure is untested. The positive predictive accuracy of PET and thallium-201 to identify hibernating myocardium decreases in parallel with worsening left ventricular function..$^{578}$ Dobutamine echocardiography, which is the least expensive and most widely available technique, appears to carry a higher false negative rate than thallium201 scintigraphy and PET in patients with severe left ventricular dysfunction and heart failure, ${ }^{89}$ so that although a positive dobutamine echocardiography result provides good evidence of viability, a second investigation may be required before a patient with a negative test can be considered unlikely to benefit from revascularisation.

As expected, there is a consistent relation between the amount of viable myocardium and the improvement in left ventricular function following revascularisation. ${ }^{35} \mathrm{~A}$ number of small studies have indicated that to obtain a significant improvement in left ventricular function, $>50 \%$ of the ventricular myocardium needs to show convincing evidence of viability. ${ }^{35}$

\section{What is the risk of revascularisation in heart} failure?

The decision to revascularise must balance the risk of myocardial damage at the time of CABG against the benefit of revascularising hibernating segments. Low ejection fraction and worse functional state independently increase the risk of CABG. Earlier series of CABG in patients with poor ventricular function demonstrated a high mortality $(11-16 \%) .{ }^{10-12}$ More recently, in highly selected patients, perioperative mortality rates were between $2.1 \%$ and $6.6 \%,{ }^{313-15}$ presumably as a result of advances in the identification of hibernating myocardium and improvements in anaesthetic, surgical, and myocardial protection techniques. In these series, although all patients had poor ventricular function (mean (SD) ejection fraction 22 (3)\%) the percentage with severe heart failure symptoms (New York Heart Association (NYHA) $\geqslant$ III) varied from $24 \%{ }^{15}$ to $100 \%{ }^{3}{ }^{14}$ It has become apparent that lack of substantial myocardial viability confers a high operative risk, ${ }^{3}{ }^{16}$ and that in such highly selected patients, very few ventricular segments deteriorate following CABG. ${ }^{3}$ Nevertheless, the enthusiasm of surgeons to perform what remains comparatively high risk surgery may be dampened if non-risk stratified surgical results are used as key performance indicators. 
What benefits of revascularisation can be expected? The old randomised trials of coronary surgery excluded patients with heart failure symptoms (NYHA > II) and the coronary artery surgery study (CASS) registry comparing the survival of patients with predominant symptoms of heart failure treated medically and surgically showed an equally poor five year survival rate of $23 \%$ for both groups. ${ }^{17}$ A number of studies from the mid-1980s (antedating the recognition of myocardial hibernation) reported a survival advantage of surgical revascularisation in patients with severe left ventricular dysfunction and predominant angina. ${ }^{17-19}$ In these reports the 5-7 year survival of the surgically treated groups ranged between $63 \%$ and $69 \%$ and that of the medically treated groups between $34 \%$ and $43 \%$. However, these reports are retrospective and non-randomised ${ }^{17-19}$ and biased by the inclusion in the surgical groups of patients with more severe angina, more extensive but operable coronary artery disease, and less congestive heart failure, while patients with severe congestive heart failure tended to be treated medically.

Although we now have the ability to detect hibernating myocardium with reasonable accuracy, no controlled study of the effects of revascularisation on patients with viable myocardium has been performed. There are data from uncontrolled series, which have examined outcomes such as left ventricular function, exercise tolerance, and quality of life following CABG in patients with severe heart failure, even in the absence of angina or exercise inducible ischaemia. ${ }^{31420}$ In general, the results have been encouraging with substantial improvements in left ventricular function, ${ }^{351314}$ exercise tolerance, ${ }^{320}$ and quality of life. ${ }^{3}$ Another alleged benefit of revascularising hibernating myocardium is a favourable influence on the remodelling process of non-ischaemic remote myocardial regions, perhaps reducing subsequent hypertrophy and dilatation.

Heart failure caused by ischaemic heart disease?

If yes $\checkmark$

Are the coronary arteries amenable to revascularisation?

If yes

Are there dysfunctional myocardial segments subtended by flow-limiting coronary lesions?

If yes

Are these segments viable (dobutamine echocardiography, thallium scintigraphy, PET)?

If yes

Are there sufficient segments to indicate that a clinically
significant improvement may occur after revascularisation?

If yes

Operate

Figure 1 Possible algorithm to determine which heart failure patient would benefit from revascularisation.

\section{Summary}

Although ischaemic heart disease is the most common cause of heart failure in the developed world, optimal treatment remains unclear. Medical treatment remains limited in its effectiveness with persistently high mortality and morbidity rates, and transplantation is an option available to very few. Revascularisation aimed at improving left ventricular function rather than relief of angina is a promising option that should be properly tested. A prospective randomised study comparing revascularisation with optimal medical treatment in patients with ischaemic heart failure but viable myocardium is required. Until the results of such a study become available cardiologists wishing to consider revascularisation for their heart failure patients might consider the algorithm (fig 1).

D PAGANO

J N TOWNEND

R S BONSER

Departments of Cardiology and Cardiothoracic Surgery,

Queen Elizabeth Hospital and University of Birmingham,

Birmingham B15 2TH, UK

email:j.n.townend@bham.ac.uk

1 Arnese M, Cornel J, Salustri A, et al. Prediction of improvement of regional left ventricular function after surgical revascularisation. A comparison of low-dose dobutamine echocardiography with 201-Tl single photon emission computed tomography. Circulation 1995;91:2748-52.

2 Bax J, Cornel J, Visser F, et al. Prediction of recovery of myocardial dysfunction after revascularization. Comparison of fluorine-18 fluorodeoxyglucose/thallium SPECT, thallium-201 stress-reinjection and dobutamine glucose/thallium SPECT, thallium-201 stress-reinjection
echocardiography. F Am Coll Cardiol 1996;28:558-64.

3 Pagano D, Townend J, Horton R, et al. Coronary artery bypass grafting for ischemic heart failure. The predictive value of quantitative PET for sympischemic heart failure. The predictive value of quantitative PET for symp-
tomatic and functional outcome. $\mathcal{F}$ Thorac Cardiovascul Surg 1998;115:791-

4 Wijns W, Vatner S, Camici PG. Hibernating myocardium. N Engl f Med 1998;339:173-81.

5 vom Dahl J, Eitzman DT, al Aouar ZR, et al. Relation of regional function, perfusion, and metabolism in patients with advanced coronary artery disease undergoing surgical revascularization. Circulation 1994;90:235666.

6 Bonow R. Identification of viable myocardium. Circulation 1996;94:2674-8.

7 Fath-Ordoubadi F, Pagano D, Marinho N, et al. Coronary revascularisation in the treatment of moderate and severe postischemic left ventricular dysfunction. Am 7 Cardiol 1998;82:26-31.

8 Perrone-Filardi P, Pace L, Prastraro M, et al. Assessment of myocardial viability in patients with chronic coronary artery disease. Rest-4-hour-24hour 201-thallium tomography versus dobutamine echocardiography. Circulation 1996;94:2712-19.

9 Pagano D, Bonser RS, Townend J, et al. Predictive value of dobutamine echocardiography and positron emission tomography in identifying hibernating myocardium in patients with postischaemic heart failure. Heart hibernating myocar

10 Louie H, Laks H, Milgalter E, et al. Ischaemic cardiomyopathy. Criteria for myocardial revescularisation and cardiac transplantation. Circulation 1991; 86(suppl III):III-290-5.

11 Luciani GB, Faggian G, Razzolini R, et al. Severe ischaemic left ventricular failure: coronary operation or heart transplantation? Ann Thorac Surg 1993;55:719-23.

12 Milano CA, White WD, Smith LR, et al. Coronary artery bypass in patients with severely depressed ventricular function. Ann Thorac Surg 1993;56: 487-93.

13 Gunning $M$, Chua T, Harringhton D, et al. Hibernating myocardium: clini$\mathrm{cal}$ and functional response to revascularisation. Eur $\mathcal{F}$ Cardiothorac Surg 1997;11:1105-12.

14 Dreyfus G, Duboc D, Blasco A, et al. Myocardial viability assessment in ischaemic cardiomyopathy: benefits of coronary revascularisation. Ann Thorac Surg 1994;57:1402-8.

15 Mickleborough L, Maruyama H, Tagaki T, et al. Results of revascularisation in patients with severe left ventricular dysfunction. Circulation 1995; 92(suppl II):II-73-9.

16 Pagley P, Beller G, Watson D, et al. Improved outcome after coronary artery bypass surgery in patients with ischemic cardiomyopathy and residual myocardial viability. Circulation 1997;96:793-800.

17 Alderman E, Fisher L, Litwin P, et al. Results of coronary artery surgery in patients with poor left ventricular function (CASS). Circulation 1983;74: 785-95.

18 Bounous EP, Mark DB, Pollock BG, et al. Surgical survival benefit for coronary disease patients with left ventricular dysfunction. Circulation 1988;78(suppl I):I-151-7

19 Pigott JD, Kouchoukos NT, Oberman A, et al. Late results of surgical and medical therapy for patients with coronary artery disease and depressed left ventricular function. $7 \mathrm{Am}$ Coll Cardiol 1985;5:1036-45.

20 Di Carli M, Asgarzadie F, Schelbert H, et al. Quantitative relation between myocardial viability and improvement in heart failure symptoms after revascularisation in patients with ischaemic cardiomyopathy. Circulation 1995;92:3436-44. 